\title{
Nicht nur wehrlose Sündenböcke! Schuldvermeidungsstrategien internationaler Organisationen
}

\author{
Tim Heinkelmann-Wild $(\mathbb{D} \cdot$ Bernhard Zangl
}

Online publiziert: 18. Juni 2020

(C) Der/die Autor(en) 2020

Zusammenfassung Wie reagieren internationale Organisationen (IOs) auf Schuldzuweisungen ihrer Mitgliedstaaten? Oftmals werden in der Forschung IOs im Falle von gescheiterten Politiken als gute Sündenböcke für die Schuldzuweisungen ihrer Mitgliedstaaten gesehen, weil sie sich kaum zu Wehr setzen müssen, können oder wollen. Demgegenüber argumentieren wir, dass IO-RepräsentantInnen versuchen, IOs in der Öffentlichkeit nicht als Schuldige für gescheiterte Politiken dastehen zu lassen. IOs sind deshalb keine passiven ,blame taker“, sondern aktive „blame avoider". In manchen Fällen sind sie zwar bereit, die Schuldzuweisungen ihrer Mitgliedstaaten zu ignorieren, doch in anderen Fällen versuchen sie ihre Verantwortung zu verschleiern oder sogar die Mitgliedstaaten zu attackieren. Um dies zu erklären, entwickeln wir eine Theorie der Schuldvermeidung von IOs, gemäß derer die Autorität einer IO bestimmt, welche Schuldvermeidungsstrategie ihre RepräsentantInnen einschlagen. Je nachdem, ob eine IO im betreffenden Politikfeld Autorität intergouvernemental, supranational oder hybrid ausübt, wird sie auf Schuldzuweisungen ihrer Mitgliedstaaten durch Strategien des Ignorierens, Verschleierns oder Attackierens reagieren. Wir demonstrieren die Plausibilität unserer Theorie mithilfe einer Medieninhaltsanalyse der Verantwortungsattributionen der Europäischen Kommission für drei umstrittene EU-Migrationspolitiken. Wir tragen damit zum besseren Verständnis der öffentlichen Schuldvermeidungsstrategien von IOs bei.

Schlüsselwörter Europäische Kommission · Europäische Union · Internationale Autorität · Schuldzuweisungen · Verantwortungsattribution

Zusatzmaterial online Zusätzliche Informationen sind in der Online-Version dieses Artikels (https:// doi.org/10.1007/s11615-020-00255-1) enthalten.

T. Heinkelmann-Wild $(\bowtie) \cdot$ B. Zangl

Geschwister-Scholl-Institut für Politikwissenschaft, Lehrstuhl für Global Governance und

Public Policy, Ludwig-Maximilians-Universität München, Oettingenstraße 67, 80538 München,

Deutschland

E-Mail: tim.heinkelmann-wild@gsi.lmu.de 


\title{
No Defenseless Scapegoats! Blame Avoidance Strategies of International Organizations
}

\begin{abstract}
How do international organizations (IOs) respond to their member states' blame attributions? Research often depicts IOs as good scapegoats for their member states when IO policies fail because they do not have to and are unable and unwilling to defend themselves. On the contrary, we argue that IO representatives try to prevent IOs from taking the blame for failed policies in public. IOs are thus not passive blame takers, but active blame avoiders. In some cases, they are willing to ignore the attribution of blame by their member states, but in other cases they try to blur their own responsibility or even attack the member states. To explain this, we develop a theory of blame avoidance by IOs according to which the authority of an IO determines which blame avoidance strategy its representatives adopt. Depending on whether the authority an IO exercises is intergovernmental, supranational or hybrid in the respective policy field, it will react to blame attributions from its member states through strategies of ignoring, blurring or attacking. We demonstrate the plausibility of our theory by means of a media content analysis of the European Commission's responsibility attributions for three contested EU migration policies. We thereby contribute to a better understanding of IOs' blame avoidance strategies.
\end{abstract}

Keywords Blame attribution · European Commission · European Union · International authority $\cdot$ Responsibility attribution

\section{Einleitung: Schuldvermeidung durch IOs}

Wenn die Politiken von internationalen Organisationen (IOs) die gewünschten Konsequenzen haben, beanspruchen die Regierungen der Mitgliedstaaten diese Erfolge gerne für sich. Sie betreiben ,credit claiming“. Scheitern IO-Politiken hingegen, so versuchen die Regierungen der Mitgliedstaaten typischerweise die Verantwortung auf IOs abzuwälzen. Sie üben sich in „blame shifting“. So wird dem Internationalen Währungsfonds (IWF) die Schuld für so manche ökonomische Rezession zugeschoben (Alcañiz und Hellwig 2011), den Vereinten Nationen (UN) die für gescheiterte Friedensmissionen (Capelos und Wurzer 2009) und der Europäischen Union (EU) die für die so genannte Flüchtlingskrise (Heinkelmann-Wild et al. 2020b).

Vor diesem Hintergrund untersucht ein rasch wachsender Forschungsstrang die Schuldabwälzung bzw. Verantwortungszuweisung von Regierungen an IOs, insbesondere die EU. ${ }^{1}$ Diese Forschung analysiert, wann Regierungen Schuld auf IOs abzuwälzen versuchen und wann IOs tatsächlich öffentlich die Verantwortung für bestimmte Politiken zugewiesen bekommen. Dabei geht sie - manchmal implizit,

\footnotetext{
1 Gramberger und Lehmann (1995); Brändström und Kuipers (2003); Gerhards et al. (2009); Hood (2011); Greuter (2014); Hobolt und Tilley (2014); Vasilopoulou et al. (2014); Sommer et al. (2016); Rittberger et al. (2017); Roose et al. (2017, 2018); Schlipphak und Treib (2017); Heinkelmann-Wild et al. (2018); Heinkelmann-Wild und Zangl (2019); Kleinen-von Königslöw et al. (2019); Sommer (2019); Traber et al. (2019); Heinkelmann-Wild et al. (2020b).
} 
manchmal aber auch explizit - davon aus, dass IOs gute Sündenböcke sind, weil sich deren führende RepräsentantInnen weniger zur Wehr setzen müssen, können und wollen als die Regierungen ihrer Mitgliedstaaten (Gramberger und Lehmann 1995; Gerhards et al. 2009; Heinkelmann-Wild und Zangl 2019; Kleinen-von Königslöw et al. 2019). Sie müssen sich weniger zur Wehr setzen, weil sie sich nicht demokratischen Wahlen zu stellen haben. Sie können sich weniger zur Wehr setzen, weil sie in der Öffentlichkeit weniger Gehör finden. Sie wollen sich weniger zur Wehr setzen, weil sie von den Regierungen der Mitgliedstaaten abhängen und also Rücksicht nehmen müssen. Mit Blick auf die EU konstatieren Gerhards, Offerhaus und Roose (2005, S. 9) daher: „Die Europäische Union [...] erfüllt [...] eine ideale Sündenbockfunktion."

Während wir im Prinzip zustimmen, dass IOs - also ihre führenden RepräsentantInnen - auf Schuldzuweisungen oftmals weniger reagieren können, wollen und müssen als dies Staaten - also deren Regierungen - üblicherweise tun, so ist doch zu erkennen, dass IOs Schuldzuweisungen nicht beliebig auf sich zu nehmen gewillt sind. Zumindest für IOs mit einem Mindestmaß an Autorität gilt - so unsere Annahme - der politische Imperativ der Schuldvermeidung. Denn mit wachsender Autorität werden sie einerseits zu einem zunehmend attraktiven Ziel staatlicher Schuldzuweisungen, sind aber andererseits auch zunehmend mehr in der Lage, darauf zu reagieren. Insofern sie diese ,scope condition“ erfüllen, sind IOs also keineswegs mehr nur passive ,blame taker“, sondern vielmehr auch aktive „blame avoider". Die Frage ist freilich, wie sie Schuldvermeidung betreiben. Wann halten sie still und ignorieren die Schuldzuweisung durch die Regierungen ihrer Mitglieder? Wann lenken sie von der eigenen Schuld ab, indem sie diese verschleiern? Und wann schlagen sie zurück, um ihrerseits die Regierungen ihrer Mitgliedstaaten zu attackieren? Kurzum: Wann entscheiden sie sich im Rahmen der Schuldvermeidung für eine Strategie des Ignorierens, des Verschleierns oder des Attackierens?

Um diese Fragen zu beantworten, entwickeln wir eine Theorie der Schuldvermeidung durch IOs, mithin ihrer führenden RepräsentantInnen. Diese Theorie nimmt an, dass die Schuldvermeidungsstrategie, die IOs wählen, um sich gegen Schuldzuweisungen von Mitgliedstaaten zu wehren, vornehmlich von der Autorität der jeweiligen IO abhängt. Danach werden RepräsentantInnen von IOs mit intergouvernementaler Autorität Schuldzuweisungen zumeist ignorieren; VertreterInnen von IOs mit supranationaler Autorität werden Schuld verschleiern; und die RepräsentantInnen von IOs, die sich Autorität mit den Mitgliedstaaten teilen, werden ihre Mitgliedstaaten attackieren. Denn je nach Autorität der IO - intergouvernemental, supranational oder hybrid - stellen sich für ihre führenden RepräsentantInnen einerseits die Notwendigkeiten und andererseits die Möglichkeiten, gegen mitgliedstaatliche Schuldzuweisungen vorzugehen, unterschiedlich dar.

Um diese These zu stützen, geht das Papier folgendermaßen vor: In einem ersten Schritt entwickeln wir die angedeutete Theorie der Schuldvermeidung von IOs (Abschn. 2). In einem zweiten Schritt legen wir das Forschungsdesign dar, im Rahmen dessen wir die These unserer Theorie empirisch anhand der öffentlichen Reaktionen der Europäischen Kommission auf Schuldzuweisungen der EU-Mitgliedstaaten für drei umstrittene EU-Migrationspolitiken plausibilisieren wollen (Abschn. 3). In einem dritten Schritt zeigen wir, dass die EU-Kommission auf mitgliedstaatliche 
Schuldzuweisungen in den Fällen (1) des oft gescholtenen Gemeinsamen Europäischen Asylsystems, (2) der umstrittenen Politik zum Schutz der EU-Außengrenzen und (3) des kritisierten EU-weiten Anspruchs auf Sozialleistungen wie von unserer Theorie erwartet reagiert (Abschn. 4). Abschließend diskutieren wir die Implikationen unserer Ergebnisse für die Forschung von Schuldzuweisungen in IOs im Allgemeinen (Abschn. 5).

\section{Theorie: Schuldvermeidungsstrategien von IOs}

Wenn Regierungen von Mitgliedstaaten IOs für problematische Politiken verantwortlich machen, dann kann dies nicht nur die Legitimität ihrer Politiken, sondern auch ihre Legitimität insgesamt und damit ihre politische Autorität beeinträchtigen (Kumlin 2011; Schlipphak und Treib 2017). Dies ist umso problematischer, als IOs selbst wenig Machtressourcen besitzen und somit zum Erhalt ihrer Autorität auf den Legitimitätsglauben in der Öffentlichkeit angewiesen sind (Buchanan und Keohane 2006; Tallberg et al. 2018; Tallberg und Zürn 2019). Tatsächlich beklagt etwa die Europäische Kommission (2017, S. 12) mit Blick auf die eigenen Legitimationsprobleme: „Blaming ,Brussels“ $[$...] and the habit of finger-pointing [...] have already proved damaging.“

Da die Schuldzuweisungen - oder auch Verantwortungsattributionen ${ }^{2}$ - durch Mitgliedstaaten für IOs Legitimitätskosten haben, werden führende IO-RepräsentantInnen zur Wahrung der Autorität ,ihrer" IO darauf reagieren und sich zur Wehr setzen wollen. Unter führenden IO-RepräsentantInnen verstehen wir insbesondere IO-Generaldirektoren wie den Präsidenten der Europäischen Kommission und deren engsten Führungsstab, also etwa die Kommissare der EU (Gronau und Schmidtke 2016, S. 542-543). ${ }^{3}$ Wie alle politischen RepräsentantInnen, deren Organisationen öffentlich für gescheiterte Politiken verantwortlich gemacht werden, werden auch führende IO-RepräsentantInnen versuchen, ,ihre“ IO nicht - oder zumindest nicht alleine - als Schuldige dastehen zu lassen (Weaver 1986, 2018; Hood 2011; Hood et al. 2016). Der noch jungen Forschung zur Öffentlichkeitsarbeit von IOs folgend ${ }^{4}$, nehmen wir deshalb an, dass ihre führenden RepräsentantInnen öffentlich im Namen „,ihrer“ IO strategische Schuldvermeidung - „blame avoidance“ - betreiben wollen. Darüber hinaus nehmen wir an, dass IOs zur öffentlichen Schuldvermeidung auf unterschiedliche Strategien zurückgreifen können:

\footnotetext{
${ }^{2}$ Wir definieren Schuldzuweisung als die Zuschreibung von Verantwortung für einen negativ dargestellten Sachverhalt. Schuldzuweisung ist ,the act of attributing something considered to be bad or wrong to some person or entity" (Hood 2011). Für die Zwecke dieses Beitrags verwenden wir den Begriff der Verantwortungszuschreibung, der sich auch auf positive Sachverhalten beziehen kann (Gerhards et al. 2009), als Synonym.

${ }^{3}$ Der Einfachheit halber werden wir im Folgenden teils anstatt von „führenden IO-RepräsentantInnen“ schlicht von IOs sprechen.

${ }^{4}$ Capelos und Wurzer (2009); Zaum (2013); Gronau und Schmidtke (2016); Ecker-Ehrhardt (2018a, 2018b, 2018c); Dingwerth et al. (2019a, 2019b); Rauh und Zürn (2019); Squatrito et al. (2019); Greenhill (2020); Rauh et al. (2020).
} 
(1) Ignorieren IO-RepräsentantInnen können die Schuldzuweisungen von Mitgliedstaaten ignorieren (vgl. Hood et al. 2016, S. 544; Traber et al. 2019, S. 1103-1104). Um in der Öffentlichkeit möglichst wenig als Schuldige für gescheiterte IO-Politiken dazustehen, halten sie still. Sie wollen, dass die gescheiterte IO-Politik öffentlich möglichst wenig thematisiert wird, sodass die eigene Schuld daran möglichst wenig wiegt. Um nicht Öl ins Feuer der öffentlichen Schuldzuweisungen zu gießen, werden IO-RepräsentantInnen sogar auf jede Relativierung oder Rechtfertigung der gescheiterten IO-Politik verzichten. Sie ,deal[s] with blame by saying as little as possible“ und ,sit[ting] out a blame firestorm until it passes over“ (Hood 2011, S. 59). Frei nach dem Motto „du sollst keine schlafenden Hunde wecken“ lassen sie Schuldzuweisungen ihrer Mitglieder ins Leere laufen.

(2) Verschleiern IO-RepräsentantInnen können aber auch versuchen, die Schuld an einer gescheiterten IO-Politik zu verschleiern. Um in der Öffentlichkeit nicht alleine als Schuldiger dazustehen, zielen sie auf eine ,diffusion of responsibility across multiple actors“ (Hobolt und Tilley 2014, S. 106; vgl. Weaver 1986, S. 385). Die Verantwortung ihrer IO durchaus konzedierend werden sie die Mitverantwortung von Drittparteien, einschließlich ihrer Mitgliedstaaten, hervorheben. Sie werden die eigene Schuld in der Öffentlichkeit relativieren und die gescheiterte Politik rechtfertigen, zum Beispiel indem sie auf widrige Umstände verweisen. Frei nach dem Motto ,mitgegangen, mitgefangen“ werden sie die Mitgliedstaaten mit in die Verantwortung nehmen.

(3) Attackieren IO-RepräsentantInnen können Schuld zudem vermeiden, indem sie die Mitgliedstaaten ihrerseits durch klare Schuldzuweisung attackieren. Indem sie diese in der Öffentlichkeit direkt beschuldigen, versuchen sie von der Schuld der IO abzulenken (vgl. Weaver 1986, S. 385, 2018; Hobolt und Tilley 2014, S. 102-103; Gerhards et al. 2009; Heinkelmann-Wild und Zangl 2019). Die IO-RepräsentantInnen mögen hier zwar anerkennen, dass die betreffende IO-Politik gescheitert ist, weisen aber öffentlich jede Schuld ganz unmissverständlich von sich und ihrer IO. Vielmehr nutzt sie jede Gelegenheit, um die Schuld der Mitgliedstaaten für die betreffende Politik öffentlich zu unterstreichen. Frei nach dem Motto „Angriff ist die beste Verteidigung“ geht die IO zum offenen Gegenangriff auf ihre Mitgliedstaaten über.

Welche der drei Schuldvermeidungsstrategien IOs einsetzen hängt aus unserer Sicht von der Autorität der jeweiligen IO ab (siehe Abb. 1). IOs besitzen Autorität in dem Maße, in dem ihr Anspruch, verbindliche Entscheidungen zu treffen und umsetzen zu können, von ihren Adressaten anerkannt wird (vgl. Zürn et al. 2012, S. 70; Abbott et al. 2020, S. 12). In diesem Sinne - und nicht nur formalrechtlich - unterscheiden wir zwischen intergouvernementaler, supranationaler und hybrider IO-Autorität, je nachdem, ob ihr Anspruch anerkannt wird, Entscheidungen über und die Umsetzung von IO-Politiken unabhängig von den Regierungen der Mitgliedstaaten bestimmen zu können (Hooghe und Marks 2015; Rittberger et al. 2017). RepräsentantInnen von IOs mit intergouvernementaler Autorität werden eher eine Strategie des Ignorierens wählen, die von IOs mit supranationaler Autorität vor- 


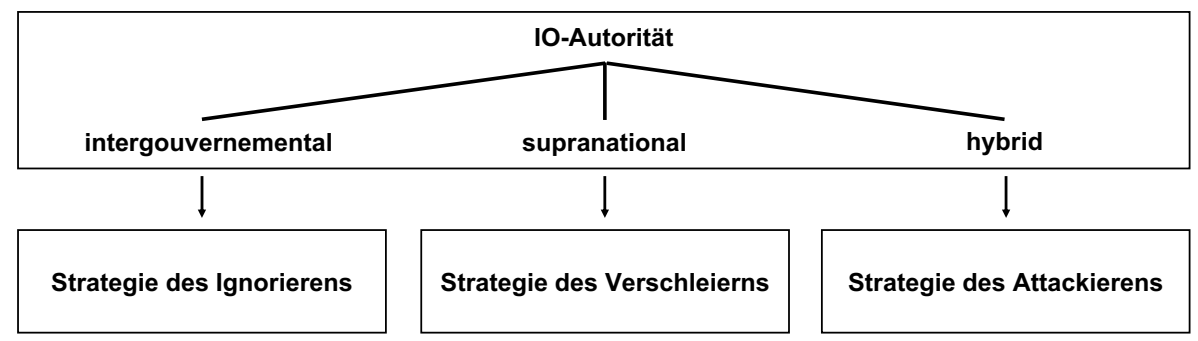

Abb. 1 Die Autorität und die Schuldvermeidungsstrategien von internationalen Organisationen (IOs)

nehmlich auf eine Strategie des Verschleierns setzen; und die von IOs mit hybrider Autorität wählen zumeist eine Strategie des Attackierens. Denn je nach Autorität der IO können ihre führenden Vertreter die Schuld der IO für fehlgeschlagene Politiken mehr oder weniger plausibel bestreiten.

Die ,plausible deniability“ - die plausible Bestreitbarkeit - von Schuld ist für die Schuldvermeidungsstrategien von IOs wichtig, obwohl die Öffentlichkeit oftmals zunächst wenig über die Autoritätsstrukturen in IOs weiß und mithin kaum einschätzen kann, welche Entscheidungs- bzw. Umsetzungskompetenzen sie haben (vgl. Hobolt und Tilley 2014, S. 45; Wilson und Hobolt 2015; Rittberger et al. 2017, S. 912; León et al. 2018, S. 661). Denn wenn Staaten IOs die Schuld für fehlgeschlagene Politiken zuschreiben, dann setzt zumeist ein öffentlicher Diskurs ein, welcher gerade die Autoritätsstrukturen der IO transparent macht. Und dies gilt insbesondere dann, wenn IOs die Schuldzuweisungen nicht nur passiv hinnehmen, sondern ihnen aktiv begegnen. Die Öffentlichkeit wird im Diskurs über die Autoritätsstrukturen der betreffenden IO sukzessive aufgeklärt. Die aktuelle Debatte über die Mitverantwortung der Weltgesundheitsorganisation (WHO) an der Coronapandemie mag dies verdeutlichen. Seit US-Präsident Donald Trump fehlerhafte WHO-Politiken für die Coronakrise verantwortlich gemacht hat, wird die Öffentlichkeit durch diese Debatte nicht zuletzt über die Autorität der WHO aufgeklärt. Um die Plausibilität der Schuldzuweisungen zu prüfen, werden die Entscheidungsund Umsetzungskompetenzen der WHO beleuchtet.

Da die Führungsebene von IOs diesen öffentlichen „Aufklärungsprozess“ zumeist antizipieren wird, wird sie ihre Schuldvermeidungsstrategie von vornherein von der eigenen Autorität - sowohl ihrer Entscheidungs- als auch ihrer Umsetzungsautorität - abhängig machen. Sie wird darauf achten, dass ihre Schuldzuweisungen angesichts der Autorität der IO nicht als unplausibel dargestellt werden können. Um bei der öffentlichen Schuldvermeidung den Anschein von Wahrhaftigkeit (Kunda 1990, S. 482-483) zu wahren, wird sie eine Schuldvermeidungsstrategie wählen, die ihre eigene Autorität - ihre Entscheidungs- und Umsetzungsautorität - in Rechnung stellt. Sie wird je nach Autorität der IO unterschiedliche Notwendigkeiten und auch unterschiedliche Möglichkeiten der öffentlichen Schuldvermeidung sehen. Deshalb wird sich - so unsere Annahme - ihre Schuldvermeidungsstrategie an diesen Notwendigkeiten und auch Möglichkeiten ausrichten (vgl. Hood 2011, S. 76, 146-147; Schwarzenbeck 2015, S. 37; Heinkelmann-Wild und Zangl 2019; HeinkelmannWild et al. 2020b): 
(1) Intergouvernementale Autorität Bei IOs mit lediglich intergouvernementaler Autorität sind die Möglichkeiten, mitgliedstaatlichen Schuldzuweisungen in der Öffentlichkeit zu entrinnen, vergleichsweise gut. ${ }^{5}$ Da in intergouvernementalen IOs alle relevanten Entscheidungen durch die Mitgliedstaaten getroffen werden, die dann auch für deren Umsetzung verantwortlich sind, können ihre führenden RepräsentantInnen stets plausibel argumentieren, dass ihre IO für die gescheiterten Politiken überhaupt nicht verantwortlich ist. Wenn sie durch mitgliedstaatliche Schuldzuweisungen angegriffen werden, können IOs also die Schuld stets plausibel bestreiten und auf die Mitgliedstaaten abwälzen (vgl. Schwarzenbeck 2015, S. 37; HeinkelmannWild und Zangl 2019; Heinkelmann-Wild et al. 2020b).

Trotzdem ist bei IOs mit intergouvernementaler Autorität nicht zu erwarten, dass ihre RepräsentantInnen die Mitgliedstaaten offen attackieren. Da sie aufgrund intergouvernementaler Autorität von vornherein wenig plausible Adressaten derartiger Schuldzuweisungen sind, ist die Notwendigkeit dazu kaum gegeben (vgl. Schwarzenbeck 2015, S. 37; Heinkelmann-Wild und Zangl 2019; Heinkelmann-Wild et al. 2020b). Denn selbst wenn sie Schuldzuweisungen der Mitgliedstaaten ignorieren, bleibt die Gefahr gering, am Ende in der Öffentlichkeit tatsächlich als Schuldige dazustehen (vgl. Hood et al. 2016, S. 544; Traber et al. 2019, S. 1103-1104; Heinkelmann-Wild et al. 2020b). Schließlich können sie darauf vertrauen, in der Öffentlichkeit von anderen politischen Akteuren entsprechend verteidigt zu werden. Die Mitgliedstaaten durch eigene Schuldzuweisungen zu attackieren oder die eigene Schuld zu verschleiern, wäre zudem möglicherweise sogar kontraproduktiv. Denn entsprechende Schuldzuweisungen würde nicht nur die Mitgliedstaaten gegen sie aufbringen, sondern auch unnötig viel Aufmerksamkeit auf die gescheiterte IOPolitik lenken (vgl. Boin et al. 2009, S. 89; Heinkelmann-Wild et al. 2020b).

Insgesamt erwarten wir also, dass RepräsentantInnen von IOs mit vornehmlich intergouvernementaler Autorität in der Öffentlichkeit mitgliedstaatliche Schuldzuweisungen für gescheiterte IO-Politiken weitgehend ignorieren werden.

(2) Supranationale Autorität Haben IOs supranationale Autorität, so ist die Notwendigkeit für ihre RepräsentantInnen, mitgliedstaatlichen Schuldzuweisungen in der Öffentlichkeit zu begegnen, besonders groß. ${ }^{6}$ Denn anders als IOs mit intergouvernementaler Autorität verfügen IOs mit supranationaler Autorität über unabhängige politische Entscheidungs- und Umsetzungskompetenz und werden damit zu einem plausiblen Adressaten mitgliedstaatlicher Schuldzuweisungen (vgl. Schwarzenbeck 2015, S. 37; Heinkelmann-Wild und Zangl 2019; Heinkelmann-Wild et al. 2020b). Dementsprechend können IOs kaum hoffen, dass ihre Verantwortung für die gescheiterte Politik dann unbemerkt bleibt, wenn sie mitgliedstaatliche Schuldzu-

\footnotetext{
5 Die Autorität von IOs ist dann intergouvernemental, wenn die Entscheidungen über und die Umsetzung von IO-Politiken durch die Regierungen ihrer Mitgliedstaaten kontrolliert wird. In intergouvernementalen IOs haben von den Staaten unabhängige Organe wie die IO-Verwaltung keine nennenswerten Entscheidungs- oder Umsetzungskompetenzen (Hooghe und Marks 2015; Rittberger et al. 2017).

${ }^{6}$ Eine IO besitzt dann supranationale Autorität, wenn die Mitgliedstaaten die Entscheidungen über und die Umsetzung von IO-Politiken nur mehr begrenzt kontrollieren können. In supranationalen IOs haben von den Staaten unabhängige Organe wie die Verwaltung eigenständige Entscheidungs- und Umsetzungskompetenzen (Hooghe und Marks 2015; Rittberger et al. 2017).
} 
weisungen einfach ignorieren. Sie können also nicht passiv bleiben, sondern müssen sich aktiv wehren, um in der Öffentlichkeit nicht als Schuldige dazustehen (vgl. Traber et al. 2019, S. 3-4; Hood et al. 2016, S. 544).

Dabei sind allerdings für IOs mit supranationaler Autorität die Möglichkeiten, in der Öffentlichkeit mitgliedstaatlichen Schuldzuweisungen zu entkommen, besonders beschränkt. Da sie eigenständige Entscheidungs- und Umsetzungskompetenzen besitzen, können ihre führenden RepräsentantInnen in der Öffentlichkeit die IOVerantwortung für gescheiterte Politiken kaum plausibel abstreiten (vgl. Hobolt und Tilley 2014, S. 115). Insbesondere können sie die Schuld nicht einfach den Mitgliedstaaten in die Schuhe schieben (vgl. Schwarzenbeck 2015, S. 37; Heinkelmann-Wild und Zangl 2019; Heinkelmann-Wild et al. 2020b). Diese offen zu attackieren, wäre ein Schuss ins Knie (vgl. Hood et al. 2016, S. 544). Deshalb werden RepräsentantInnen von IOs mit supranationaler Autorität versuchen müssen, ihre eigene Schuld für gescheiterte IO-Politiken zu verschleiern (Weaver 1986, S. 385, 389; Hobolt und Tilley 2014, S. 113-117). Ohne die Mitgliedstaaten gegen sich aufzubringen, werden sie in der Öffentlichkeit ganz diplomatisch auf deren Mitverantwortung verweisen.

Insgesamt erwarten wir also, dass RepräsentantInnen von IOs mit eher supranationaler Autorität die Schuld für gescheiterte IO-Politiken typischerweise in der Öffentlichkeit zu verschleiern versuchen.

(3) Hybride Autorität Wird in IOs politische Autorität hybrid - also durch intergouvernementale und supranationale Organe gemeinsam - ausgeübt, so ist für IORepräsentantInnen sowohl die Notwendigkeit als auch die Möglichkeit gegeben, den Schuldzuweisungen ihrer Mitgliedstaaten für gescheiterte Politiken in der Öffentlichkeit aktiv zu begegnen. Eine hohe Notwendigkeit besteht, da unabhängige IOOrgane an der Entscheidungsfindung und der Umsetzung beteiligt sind und somit ein plausibler Adressat mitgliedstaatlicher Schuldzuweisungen sind (vgl. Schwarzenbeck 2015, S. 37; Heinkelmann-Wild und Zangl 2019; Heinkelmann-Wild et al. 2020b). Deshalb müssen IO-RepräsentantInnen damit rechnen, dass die Mitgliedstaaten versuchen werden, der IO die Schuld für ihre gescheiterte Politik in die Schuhe zu schieben. Sie werden also öffentliche Schuldzuweisungen nicht einfach ignorieren können, sondern aktiv dagegen vorgehen müssen: „Higher levels of blame will be likely to lead to active attempts to reduce or remove it than,do nothing " or ,no comment" responses" (Hood et al. 2016, S. 544; vgl. Traber et al. 2019, S. 3-4).

Zugleich aber sind die Möglichkeiten für IOs, die sich Autorität mit den Mitgliedstaaten teilen, Verantwortung an die Mitgliedstaaten zurückzuweisen, nicht schlecht. Da die Mitgliedstaaten an den Entscheidungen über und der Umsetzung von IO-Politiken beteiligt sind, können IO-RepräsentantInnen die Schuld durchaus plausibel abweisen. Um nicht allein als Schuldige dazustehen, kann die IO zumindest auf die Mitschuld der Mitgliedstaaten verweisen (vgl. Schwarzenbeck 2015, S. 37; Heinkelmann-Wild und Zangl 2019; Heinkelmann-Wild et al. 2020b). Deshalb werden RepräsentantInnen der betroffenen IO wahrscheinlich zunächst auch versuchen, die eigene Schuld lediglich zu verschleiern, indem sie die Mitgliedstaaten mit in die Verantwortung nimmt. Je mehr die IO allerdings mit mitgliedstaatlichen Schuldzuweisungen überzogen wird, desto mehr wird sie geneigt sein, ihrerseits die Mit- 
gliedstaaten durch Schuldzuweisung offen zu attackieren. Sie wird zum Gegenangriff übergehen ,[to] deflect blame by blaming others“ (Weaver 1986, S. 385).

Insgesamt erwarten wir also, dass RepräsentantInnen von IOs mit hybrider Autorität die Mitgliedstaaten attackieren, um so deren Schuldzuweisungen für gescheiterte IO-Politiken in der Öffentlichkeit zurückzuweisen.

Zusammengefasst gehen wir also von einem kurvilinearen Zusammenhang zwischen der Autorität einer IO und der ihrer Schuldvermeidung aus. Dort, wo IOs besonders wenig (intergouvernemental) und besonders viel (supranational) Autorität ausüben, erwarten wir vergleichsweise passive und vor allem auch diplomatische Reaktionen (Ignorieren und Verschleiern). Denn hier ist eine aggressive Schuldvermeidung entweder nicht notwendig (intergouvernemental) oder aber nicht möglich (supranational). Dort hingegen, wo ihre Autorität ein Hybrid (zwischen supranationaler und intergouvernementaler Autorität) ist, erwarten wir deutlich aggressivere Reaktionen (Attackieren). Denn hier sind aufgrund der hybriden Autorität derartige Schuldvermeidungsstrategien ähnlich notwendig wie bei supranationaler Autorität, aber auch ähnlich gut möglich wie bei intergouvernementaler Autorität.

\section{Forschungsdesign: EU Schuldvermeidungsverhalten im Vergleich}

Um unsere Theorie einer ersten empirischen Plausibilitätsprüfung zu unterziehen, untersuchen wir die Schuldvermeidungsstrategien führender RepräsentantInnen der EU in der EU-Migrationspolitik. Konkret vergleichen wir die Schuldvermeidungsstrategien der Europäischen Kommission bei drei EU-Migrationspolitiken, bei denen ihre Mitgliedstaaten versucht haben, der EU die Schuld für migrationspolitische Problemlagen in die Schuhe zu schieben. Unser Fokus liegt auf der EU, weil hier unsere „scope condition“ eines Mindestmaßes an IO-Autorität zweifelsfrei gegeben ist. Einerseits hat die EU im Gegensatz zu manch anderer IO genug Autorität, sodass ihr die Mitgliedstaaten im Prinzip mit einem Mindestmaß an Plausibilität Schuld zuweisen können (Hooghe und Marks 2015). Und andererseits besitzt die EU im Gegensatz zu anderen IOs (Ecker-Ehrhardt 2018a, 2018c; Kleinen-von Königslöw et al. 2019) zweifelsfrei die notwendigen Kapazitäten, um in der Öffentlichkeit strategisch zu kommunizieren (Gramberger 1997; Hoesch 2003; Gerhards et al. 2009, S. 538-539; siehe auch Jankauskas und Eckhard 2019). In der EU sollten sich Schuldvermeidungspolitiken also besonders gut beobachten lassen. ${ }^{7}$

Darüber hinaus liegt unser Fokus aber insbesondere deshalb auf der EU, weil sie im Gegensatz zu den meisten anderen IOs in unterschiedlichen Politikbereichen über unterschiedliche Autorität verfügt. Damit können wir im Rahmen eines Most-similar-case-Designs (Przeworski und Teune 1982, S. 32-33) besonders gut prüfen, ob sich diese Autoritätsdifferenzen, wie von uns erwartet, in gänzlich unterschiedliche Schuldvermeidungsstrategien führender RepräsentantInnen der EU -

\footnotetext{
7 In der Tat hat die jüngere Forschung gezeigt, dass über Aussagen der Europäischen Kommission und ihrer Kommissare vergleichsweise prominent in den europäischen Medien berichtet wird (Koopmans 2007; Adam und Pfetsch 2009; Gattermann 2018).
} 
also insbesondere ihrer Kommissare - übersetzen. Damit machen wir zwar - wie bei allen (quasi-)experimentellen Designs - bei der externen Validität Abstriche, können im Gegenzug aber die interne Validität unserer Ergebnisse stützen. Denn durch den Vergleich innerhalb der EU kontrollieren wir für zahlreiche Störvariablen, die einen Vergleich über verschiedene IOs hinweg mit je unterschiedlichen Autoritätsstrukturen beeinträchtigen würden.

Der Versuch, Störvariablen im Rahmen eines Most-similar-case-Designs möglichst konstant zu halten, begründet auch den Fokus auf die Migrationspolitik der EU. Indem wir das Schuldvermeidungsverhalten der Kommission (1) bei der EUAsylpolitik, (2) beim EU-Grenzschutz und (3) bei den EU-Sozialleistungen untersuchen, können wir politikfeldspezifische Unterschiede reduzieren, welche einen aussagekräftigen Vergleich stören. Immerhin beruhen sie allesamt auf denselben Grundsätzen eines freien Verkehrs über die Binnengrenzen und der gemeinsamen Sicherung der EU-Außengrenzen:

- Die Asylpolitik der EU legt fest, welches EU-Mitglied für die Bearbeitung von Asylanträgen zuständig ist. Eckpfeiler ist das sogenannte Dublin-System, das vorsieht, dass derjenige Mitgliedstaat, in dem ein Asylbewerber erstmals in die EU einreist, für dessen Asylantrag verantwortlich ist (Lavenex 2015, S. 381).

- Die Grenzschutzpolitik der EU sieht eine gemeinsame Kontrolle ihrer Außengrenzen vor. Mit Frontex ist eine eigene Agentur mit der Grenzsicherung beauftragt. Diese soll mitgliedstaatliche Grenzschutzmaßnahmen unterstützen bzw. koordinieren (Lavenex 2015, S. 381).

- Nach dem Binnenmarktprogramm der EU können ihre Mitgliedstaaten Sozialleistungen nicht grundlos auf ihre eigenen Staatsangehörigen beschränken (Leibfried 2015, S. 281). Vielmehr müssen sie diese Sozialleistungen BürgerInnen anderer Mitgliedstaaten diskriminierungsfrei gewähren.

Wie für ein Most-similar-case-Design entscheidend, unterscheiden sich die drei Migrationsfälle mit Blick auf die theoretisch interessierende Erklärungsvariable für die Schuldvermeidungsstrategien von IOs, nämlich ihrer jeweiligen Autorität. Unsere Definition von IO-Autorität schließt die Anerkennung sowohl von Entscheidungsals auch Umsetzungskompetenzen ein. Zwar ist die Entscheidungsautorität in allen drei Fällen insofern hybrid, als jeweils der Rat unter Beteiligung des Europaparlaments auf Vorschlag der Europäischen Kommission über die jeweiligen Politiken entscheiden konnte. Die Umsetzungsautorität unterscheidet sich jedoch von Fall zu Fall:

- Die Umsetzung der Asylpolitik erfolgt anerkanntermaßen durch die Mitgliedstaaten. Durch die EU - ihr Dublin-System - wird lediglich festgelegt, welches EU-Mitglied für die Bearbeitung von Asylanträgen zuständig ist (Lavenex 2015, S. 381). Die Asylverfahren selbst bleiben aber vollständig in der Hand der Mitgliedstaaten. Die Autorität der EU zeigt im Asylbereich insgesamt stark intergouvernementale Züge.

- Die Umsetzung der Grenzschutzpolitik wird hingegen anerkanntermaßen vornehmlich durch EU-Akteure getragen. Denn mit Frontex ist eine eigene Agentur damit beauftragt, einerseits Grenzschutzoperationen - etwa im Mittelmeer - 
durchzuführen und andererseits mitgliedstaatliche Grenzschutzmaßnahmen zu unterstützen bzw. zu koordinieren (Lavenex 2015, S. 381). Damit trägt die EUAutorität im Grenzschutzbereich supranationale Züge.

- Die Politik der Sozialleistungen in der EU erfordert keine aktive Umsetzung - weder durch die EU noch ihre Mitgliedstaaten. Die Mitgliedstaaten werden lediglich aufgefordert, Sozialstaatsansprüche diskriminierungsfrei zu gewähren (Leibfried 2015, S. 279-280). Die EU-Autorität ist also insgesamt ein Hybrid zwischen intergouvernementaler und supranationaler Autorität.

Während wir also durch diese Fallauswahl für mögliche Störvariablen kontrollieren, ermöglicht es uns die Varianz der Autoritätsstrukturen über die drei Fälle hinweg deren Wirkung auf die Schuldvermeidungsstrategien der Europäischen Kommission zu untersuchen. Damit können wir unsere Theorie im Rahmen eines Most-similarcase-Designs einer Plausibilitätsprobe unterziehen. Im Unterschied zu einer bloßen Illustration gehen wir dabei ein gewisses Falsifikationsrisiko ein, weil wir die Fälle nicht so gewählt haben, dass sich die Theorie automatisch bestätigt. Wir können allerdings insofern auch keinen echten Test der Theorie vorweisen, als aufgrund der Fallauswahl lediglich interne, nicht aber externe Validität beansprucht werden kann und somit Inferenzschlüsse über die gewählten Fälle hinaus schwierig bleiben (Heupel und Zangl 2004, S. 347-349).

Um die verschiedenen Schuldvermeidungsstrategien - Ignorieren, Verschleiern, Attackieren - operational zu erfassen, untersuchen wir in den drei Fällen wie die Kommission Verantwortung attribuiert, nämlich wie oft (Häufigkeit), an wen (Richtung) und wie kämpferisch (Tonlage):

- Ignorieren: Diese Strategie ist daran zu erkennen, dass Schuldzuweisungen erstens in ihrer Häufigkeit vergleichsweise selten bleiben, dann zwar zweitens in ihrer Richtung klar auf die Mitgliedstaaten zielen, dabei aber drittens eine diplomatische Tonlage pflegen.

- Verschleiern: Diese Strategie wird dadurch bemessen, dass Schuldzuweisungen erstens in ihrer Häufigkeit deutlich häufiger vorkommen und zweitens in unterschiedliche Richtungen weisen; sie schlagen dabei drittens ebenfalls einen diplomatischen Ton an.

- Attackieren: Diese Strategie erkennt man erstens an der großen Häufigkeit von Schuldzuweisungen; zweitens daran, dass diese Schuldzuweisungen in Richtung der Mitgliedstaaten zielen und dabei drittens einen eher kämpferischen Ton aufweisen.

Um die Schuldvermeidungsstrategie der EU-Kommission dementsprechend - d.h. auf der Grundlage ihres Attributionsverhaltens - bemessen zu können, haben wir eine Inhaltsanalyse der Medienberichterstattung über die drei ausgesuchten EUMigrationspolitiken durchgeführt. Dies ist in der Forschung zu Verantwortungsattributionen üblich (Gerhards et al. 2009; Rittberger et al. 2017; Heinkelmann-Wild et al. 2018, 2020a, 2020b; Heinkelmann-Wild und Zangl 2019). Konkret haben wir die Berichterstattung über die drei Migrationsfälle in neun europäischen Qualitätszeitungen im Zeitraum von Anfang 2010 bis Ende 2016 untersucht. Die Analyse beruht auf je zwei führenden Qualitätszeitungen in Österreich (Die Presse, Der 
Standard), Deutschland (Süddeutsche Zeitung, Frankfurter Allgemeine Zeitung), Irland (The Irish Times, The Irish Examiner) und Großbritannien (The Guardian, The Daily Telegraph) sowie einer Qualitätszeitung mit europaweiter Reichweite (The European Voice/Politico Europe).

Um in den genannten Qualitätszeitungen konkrete Artikel zu den drei Fällen umstrittener EU-Migrationspolitik zu identifizieren, haben wir Stichwortsuchen in digitalen Zeitungsarchiven durchgeführt (s. Onlineappendix, Tab. A.1). Da wir möglichst alle Artikel erfassen wollten, in denen sich auf unsere Fälle gerichtete Schuldzuweisungen der Europäischen Kommission finden, haben wir zunächst wenig beschränkende Suchbegriffe benutzt, um danach alle Artikel manuell auszusortieren, die sich nicht mit der jeweiligen Politik befassen oder die jeweilige Politik als gänzlich unumstritten darstellen.

In den so identifizierten insgesamt 1040 Artikeln über die drei ausgewählten EUMigrationspolitiken haben wir sodann nach Verantwortungs- bzw. Schuldzuweisungen der Europäischen Kommission gesucht. ${ }^{8}$ Dabei wurde jede Aussage der Europäischen Kommission (Attributionssender), welche die Verantwortung oder Schuld für eine der drei untersuchten Migrationspolitiken (Attributionsgegenstand) einem politischen Akteur (Attributionsadressat) zuweist, kodiert. ${ }^{9}$ Wir erfassen die Schuldvermeidungsstrategie der Europäischen Kommission, indem wir die Häufigkeit, Richtung und Tonlage dieser insgesamt 81 Attributionen in den untersuchten Fällen vergleichen. Dabei wird die Häufigkeit danach bemessen, wie viele Schuldzuweisungen der Kommission sich pro 100 Artikel finden. Die Richtung wird durch den Anteil der auf die Mitgliedstaaten gerichteten Schuldzuweisungen indiziert. Und die Tonlage haben wir über den Anteil der Schuldzuweisungen mit eindeutig kämpferi-

Tab. 1 Schuldzuweisungen der Europäischen Kommission - erwartet

\begin{tabular}{|c|c|c|c|}
\hline & $\begin{array}{c}\text { Intergouvernementale } \\
\text { IO-Autorität } \\
\text { (EU-Asylpolitik) } \\
\downarrow \\
\text { Ignorieren }\end{array}$ & $\begin{array}{c}\text { Supranationale } \\
\text { IO-Autorität } \\
\text { (EU-Grenzschutz) } \\
\downarrow \\
\text { Verschleiern }\end{array}$ & $\begin{array}{c}\text { Hybride } \\
\text { IO-Autorität } \\
\text { (EU-Sozialleistungen) } \\
\downarrow \\
\text { Attackieren }\end{array}$ \\
\hline $\begin{array}{l}\text { Häufigkeit } \\
\text { (pro } 100 \text { Artikel) }\end{array}$ & Niedrig & Hoch & Hoch \\
\hline $\begin{array}{l}\text { Richtung } \\
\text { (Anteil der Mit- } \\
\text { gliedstaaten) }\end{array}$ & Gerichtet & Ungerichtet & Gerichtet \\
\hline $\begin{array}{l}\text { Ton } \\
\text { (Anteil } \\
\text { kämpferisch) }\end{array}$ & Diplomatisch & Diplomatisch & Kämpferisch \\
\hline
\end{tabular}

IO internationale Organisation

8 Wir haben zudem in jedem der drei Fälle auch alle Schuldzuweisungen erhoben, in denen Mitgliedstaaten die EU für die entsprechenden Politiken verantwortlich machen. Denn wir wollen ja zeigen, dass die EUKommission mit ihren Schuldvermeidungsstrategien auf mitgliedstaatliche Schuldzuweisungen reagiert.

9 Wir folgen damit Gerhards et al. (2009), welche Attributionsaussagen als durch einen Attributionssender, einen Attributionsgegenstand und einen Attributionsadressaten gekennzeichnet betrachten. 
scher Ausrichtung erhoben. ${ }^{10}$ Insgesamt ergibt sich für die genannten drei Fälle der EU-Migrationspolitik die in Tab. 1 dargestellte Erwartung.

\section{Analyse: EU Schuldvermeidung in der Migrationspolitik}

Die Ergebnisse der vergleichenden Analyse der Verantwortungsattributionen der Europäischen Kommission in den untersuchten drei Fällen europäischer Migrationspolitik stimmen mit den Erwartungen unserer Theorie über das Schuldvermeidungsverhalten von IOs überein (vgl. Tab. 2).

\subsection{Die Strategie des Ignorierens in der EU-Asylpolitik}

Die Mitgliedstaaten der EU haben insbesondere im Zuge der sogenannten Flüchtlingskrise 2015 versucht, die Schuld an den offensichtlichen Defiziten der gemeinsamen Asylpolitik auf die EU abzuwälzen. Sie schoben die öffentliche Kritik an der ungleichen Lastenverteilung zwischen den Erstaufnahmeländern (im Osten und Süden der EU) und den Binnenländern (im Norden und Westen der EU) ebenso nach Brüssel weiter, wie auch die Kritik, Erstaufnahmeländern keinerlei Anreize zu bieten, die geforderte Registrierung der Flüchtlinge tatsächlich auch durchzuführen. Auch die Kritik an den erheblichen Differenzen der Asylverfahren in unterschiedlichen EU-Mitgliedstaaten wurde von den Mitgliedstaaten nach Brüssel weitergegeben. Insgesamt haben wir in den von uns analysierten 521 Medienberichten zur EUAsylpolitik 46 Aussagen gefunden (also ca. 9 pro 100 Medienberichte), in denen Mitgliedstaaten die Schuld für Missstände in der europäischen Asylpolitik der EU anlasten.

Tab. 2 Schuldzuweisungen der Europäischen Kommission - beobachtet

\begin{tabular}{lccc} 
& Intergouvernementale & Supranationale & Hybride \\
& IO-Autorität & IO-Autorität & IO-Autorität \\
& (EU-Asylpolitik) & (EU-Grenzschutz) & (EU-Sozialleistungen) \\
& $\begin{array}{l}\downarrow \\
\text { Ignorieren }\end{array}$ & Verschleiern & Attackieren \\
\hline Häufigkeit & $5(27 / 521)$ & $10(30 / 312)$ & $13(24 / 189)$ \\
(pro 100 Artikel) & Niedrig & Hoch & Hoch \\
Richtung & $100 \%(27 / 27)$ & $40 \%(12 / 30)$ & $100 \%(24 / 24)$ \\
(Anteil der Mit- & Gerichtet & Ungerichtet & Gerichtet \\
gliedstaaten) & & & $67 \%(16 / 24)$ \\
Ton & $37 \%(10 / 27)$ & $43 \%(13 / 30)$ & Kämpferisch \\
(Anteil & Diplomatisch & Diplomatisch & \\
kämpferisch) & & &
\end{tabular}

IO internationale Organisation

10 Wir definieren alle Schuldzuweisungen als im Ton kämpferisch, die von den diplomatischen Gepflogenheiten abweichen. Kritik wird hier nicht verklausuliert geäußert und Schuld wird hier nicht verklausuliert zugewiesen. Vielmehr wird der kritisierte Sachverhalt ebenso klar benannt wie der dafür Verantwortliche. 
Wie erwartet reagierte die Europäische Kommission auf die mitgliedstaatlichen Schuldzuweisungen mit einer Strategie des Ignorierens (siehe Tab. 2). Aufgrund ihrer lediglich intergouvernementalen Autorität im Bereich der Asylpolitik hatte sie zwar gute Möglichkeiten, sah aber auch nur eine geringe Notwendigkeit eine aktivere Schuldvermeidungspolitik zu betreiben. Die Häufigkeit, mit der die Europäische Kommission mit Schuldzuweisungen in den untersuchten Medienberichten auftritt, bleibt somit gerade auch im Vergleich mit dem Grenzschutz- und dem Sozialleistungsfall bemerkenswert gering (5 pro 100 Medienberichte). Die wenigen Schuldzuweisungen, die sich finden ließen, richteten sich dabei alle ausschließlich an die EU-Mitgliedstaaten (27 von 27). Und die Tonlage dieser Schuldzuweisungen bleibt wie erwartet überwiegend diplomatisch-zurückhaltend. Nur in etwa einem Drittel ihrer Verantwortungsattributionen (10 von 27) zeigte sich die Kommission klar politisch-kämpferisch.

Ein typisches Beispiel bietet der stellvertretende Kommissionspräsident Frans Timmermans, der seine Schuldzuweisung an die Mitgliedstaaten letztlich in einer Forderung, den Mitgliedstaaten zu helfen, versteckt: „We have to make sure that those countries where people arrive are better placed to make sure people are registered, that people who don't have the right to asylum are returned swiftly. ${ }^{\text {"11 }}$ Er macht damit die Mitgliedstaaten zwar insofern eindeutig verantwortlich, als er insinuiert, dass diese bei der Registrierung versagen. Aber anstatt diese Schuld ganz offen auszusprechen oder gar anzuprangern, verlangt er lediglich, dass die Mitgliedstaaten auf die Registrierung von Flüchtlingen besser vorbereitet werden müssen.

Während die Kommission das Gros ihrer Schuldzuweisung an die Mitgliedstaaten also diplomatisch verpackt, finden sich nur einige wenige Schuldattributionen, in denen sich die Kommission kämpferischer gibt. Eines der wenigen Beispiele stammt von Kommissionspräsident Jean Claude Juncker, der mit Blick auf dysfunktionale Asylsystem klagt: „Don't criticize Europe, criticize the member states.“12 Doch auch der Kommissionpräsident schlägt zumeist weniger kämpferische Töne an. Beispielsweise verpackt er seine Schuldzuweisung über die Praxis der Mitgliedstaaten, Asylsuchende unregistriert in die EU-Nachbarländer weiterreisen zu lassen, in einen Scherz: „There has been a lot of finger pointing [by Southern member states]. Not enough finger painting, but too much finger pointing in the past weeks." ${ }^{\text {"13 }}$

Insgesamt stimmen die Ergebnisse der Analyse der Schuldvermeidungspolitik der Europäischen Kommission in der EU-Asylpolitik mit unseren theoretischen Erwartungen überein. Da die EU-Autorität hier eher intergouvernementale Züge aufweist, hatte die Kommission zwar gute Möglichkeiten, sah aber auch nur eine geringe Notwendigkeit eine aktivere Schuldvermeidungspolitik zu betreiben. Sie hat sich deshalb auf eine Strategie des Ignorierens mitgliedstaatlicher Schuldzuweisungen verlagern können.

\footnotetext{
11 Frans Timmermans, Vizepräsident der EU-Kommission, zit. Weaver (2015b).

12 Jean-Claude Juncker, Präsident der EU-Kommission, zit. Baume (2015).

13 Jean-Claude Juncker, Präsident der EU-Kommission, zit. Weaver (2015a).
} 


\subsection{Die Strategie des Verschleierns in der EU-Grenzschutzpolitik}

Die Mitgliedstaaten der EU haben nicht zuletzt im Zuge der sogenannten Flüchtlingskrise 2015 versucht, der EU die Schuld an den offensichtlichen Defiziten der gemeinsamen Grenzschutzpolitik - insbesondere dem Flüchtlingsdrama mit tausenden von toten Flüchtlingen im Mittelmeer - zuzuschreiben. Egal, ob die öffentliche Kritik lautete, dass Frontex und damit die EU zu wenig tat, um die im Mittelmeer ertrinkenden Flüchtlinge zu retten, oder ob die Kritik lautete, dass Frontex und damit die EU zu viel tat, um Flüchtlinge im Mittelmeer zu retten und dadurch immer mehr Flüchtlinge ins Mittelmeer „lockte“, die Mitgliedstaaten versuchten, die Schuld an der Flüchtlingskatastrophe im Mittelmeer nach Brüssel abzuwälzen. In den von uns identifizierten 312 Medienberichten zur EU-Grenzschutzpolitik finden sich 50 derartige Schuldzuweisungen (also ca. 16 pro 100 Medienberichte).

In Übereinstimmung mit unseren theoretischen Erwartungen reagierte die Europäische Kommission auf diese Schuldzuweisungen mit einer Strategie des Verschleierns (siehe Tab. 2). Aufgrund ihrer supranationalen Autorität im Bereich des Grenzschutzes bestand zwar eine hohe Notwendigkeit, Schuld aktiv zu vermeiden. Die Häufigkeit, mit der in der untersuchten Medienberichterstattung über Schuldzuweisungen der Europäischen Kommission berichtet wird, ist hier somit deutlich höher (10 pro 100 Artikel) als im Asylfall (siehe Tab. 2). Zugleich aber sind aufgrund der supranationalen Autorität der EU ihre Möglichkeiten begrenzt, die Schuld den Mitgliedstaaten voll anzulasten. Deshalb räumt die Kommission auch die Verantwortung der EU ein und weist, anders als im Asylfall, die Schuld keineswegs nur ihren Mitgliedstaaten zu (12 von 30). Dabei wird der Ton ihrer Schuldzuweisungen zwar im Vergleich zum Asylfall etwas kämpferischer (13 von 30), bleibt aber insgesamt doch überwiegend diplomatisch-zurückhaltend.

Typisch für die Strategie des Verschleierns der Europäischen Kommission ist, dass in den Schuldzuweisungen nicht einzelne Akteure benannt werden, sondern vielmehr die EU einschließlich der Mitgliedstaaten - oder sogar Europa als Ganzes verantwortlich gemacht werden. So verkündete Kommissionspräsident José Manuel Barroso mit Blick auf das Flüchtlingsdrama im Mittelmeer: „Der Notstand von Lampedusa ist ein europäischer; Europa kann sich nicht abwenden [...] Europa kann nicht akzeptieren, dass viele tausend Menschen an seinen Grenzen umkommen." ${ }^{14}$ Auch die Hohe Vertreterin Federica Mogherini sprach vom „European conscience "15 und stellte fest: ,With this latest tragedy [...] we have no more excuses, the EU has no more excuses." ${ }^{16}$ Ähnlich bemerkte Barrosos Nachfolger Juncker: „Der Status quo ist keine Option mehr. Die gesamte $E U$ hat die moralische und humanitäre Verpflichtung zu handeln." ${ }^{17}$ Und EU-Kommissar Dimitris Avramopoulos fordert mit Blick auf das Flüchtlingsdrama im Mittelmeeer: „The European Union, its agencies

\footnotetext{
14 José Manuel Barroso, Präsident der EU-Kommission, zit. FAZ (2013); Herv. d. Autoren.

15 Federica Mogherini, EU-Kommissarin, zit. Traynor (2015); Herv. d. Autoren.

16 Federica Mogherini, EU-Kommissarin, zit. Kirchgaessner et al. (2015); Herv. d. Autoren.

17 Jean-Claude Juncker, Präsident der EU-Kommission, zit. Der Standard (2015); Herv. d. Autoren.
} 
and the member states have to step up their co-operation and common action in a spirit of solidarity and responsibility." 18

Schuldzuweisungen, die allein die Mitgliedstaaten adressieren, sind zwar im Vergleich zum Asylfall deutlich seltener, werden dabei aber im Ton ebenso selten kämpferisch. Zumeist bleiben sie diplomatisch, indem sie beispielsweise die Schuldzuweisung durch eine Forderung „tarnen“. So verlangte die EU-Außenbeauftragte Federica Mogherini: „Alle 28 Mitgliedstaaten müssen Verantwortung übernehmen; das Flüchtlingsproblem ist schon viel zu lange den südlichen Mitgliedern überlassen worden. ${ }^{\text {19 }}$ Sie insinuierte damit zwar die Schuld einiger Mitgliedstaaten an den Defiziten des EU-Grenzschutzes, doch sie verpackte dies diplomatisch in einem Appell an alle Mitgliedstaaten, die Verantwortung für eine bessere Grenzschutzpolitik gemeinsam zu übernehmen. Kämpferische Schuldzuweisungen, die die Mitgliedstaaten politisch herausfordern, sind demgegenüber sehr selten. Eines der wenigen Beispiele bietet Kommissionspräsident Juncker, der die Mitgliedstaaten mit Blick auf die toten Flüchtlinge im Mittelmeer anprangerte: „They need to do something now. I am tired of the rhetoric of consternation." ${ }^{20}$

Insgesamt decken sich die Ergebnisse der Analyse der Schuldvermeidungspolitik der Europäischen Kommission in der EU-Grenzschutzpolitik mit unseren theoretischen Erwartungen. Da die EU bei der Grenzsicherung über Autorität mit supranationalen Zügen verfügt, konnten die Mitgliedstaaten in hohem Maße Verantwortung auf die EU-Ebene schieben, was die Notwendigkeit für die Europäischen Kommission erhöhte, aus dem Fokus der Öffentlichkeit zu gelangen. Da aber ihre Möglichkeiten zugleich gering waren, die Mitgliedstaaten ihrerseits zu attackieren, verfolgte sie eine Strategie des Verschleierns.

\subsection{Die Strategie des Attackierens in der EU-Sozialleistungspolitik}

Die Mitgliedstaaten haben auch versucht, die Schuld an vermeintlichen Defiziten ihrer Politik zur Gewährung von Sozialleistungen der EU anzulasten. So schoben sie insbesondere die Kritik am sogenannten „Wohlfahrtstourismus“ bzw. dem „,Sozialstaatstourismus" nach Brüssel weiter. Danach sei die EU dafür verantwortlich, dass BürgerInnen eines Mitgliedstaats in anderen Mitgliedstaaten ansässig werden könnten, um von den dort besseren Sozialleistungen zu profitieren. Die EU zwinge sie, diesen „Sozialleistungstourismus“ zu akzeptieren. In den von uns analysierten 189 Medienberichten zu EU-Sozialleistungsansprüchen finden sich 48 derartige Anschuldigungen (also ca. 25 pro 100 Medienberichte).

Den Erwartungen unserer Theorie entsprechend, reagierte die Europäische Kommission auf diese Schuldzuweisungen mit einer Strategie des Attackierens. Denn aufgrund ihrer hybriden Autoritätsstruktur im Bereich der Sozialleistungsansprüche war einerseits die Notwendigkeit, andererseits aber auch die Möglichkeit einer aktiven Schuldvermeidungspolitik gegeben. Dementsprechend ist die Häufigkeit von Schuldzuweisung der Kommission (13 pro 100 Artikel), ähnlich wie im Grenz-

\footnotetext{
18 Dimitris Avramopoulos, EU-Kommissar, zit. Panichi (2015); Herv. d. Autoren.

19 Federica Mogherini, EU-Außenbeauftragte, zit. Straub (2015).

20 Jean-Claude Juncker, Präsident der EU-Kommission, zit. Palmeri (2015); Herv. d. Autoren.
} 
schutzfall, deutlich höher als im Asylfall (siehe Tab. 2). Dabei ist im Unterschied zum Grenzschutzfall, aber exakt wie im Asylfall, die Richtung ganz eindeutig: Alle in der Medienberichterstattung identifizierten Schuldzuweisungen richten sich an die Mitgliedstaaten (24 von 24). Und zugleich ist die Tonlage des Gros der Schuldzuweisungen ganz erheblich weniger diplomatisch als im Grenz- und im Asylfall. Die Schuldzuweisungen sind im Ton deutlich häufiger politisch-kämpferisch (16 von 24).

Ein typisches Beispiel bietet die Aussage von EU-Kommissarin Viviane Reding, welche die Mitgliedstaaten direkt attackierte, Sozialleistungen zu großzügig zu vergeben: „It seems that some national systems are too generous. Don't blame the commission or EU rules for national choices and national regulatory systems [...] Our EU rules are good and they are here to stay. Member states need to apply them to tackle abuse. "21 Und außerdem bemerkte sie:

EU rules are clear. Member states are free to decide which benefits they want to set up, under which conditions they are going to pay these, to whom and for how long. [...] EU rules do not ask member states to grant benefits unconditionally to everyone. This is the member states' choice. ${ }^{22}$

Ähnlich äußerte sich auch ihre Kommisionskollegin Vera Jourová: ,[...] member states need to tackle abuse decisively where it happens. "23 Und auch der Sprecher der Europäischen Kommission, Margaritis Schinas, bemerkte ganz unmissverständlich: „It is up to national lawmakers to fight against abuses of the system and EU law allows for this. “24 Dabei versäumte er es auch nicht, ganz undiplomatisch zu betonen, dass die Probleme des vorgeblichen Sozialleistungstourismus ,without drama and [...] calmly and carefully“ zu diskutieren wären. Und auch EU-Kommisar László Andor beschwerte sich wenig diplomatisch, dass die Debatte ,in some member states“ - gemeint waren Deutschland und das Vereinigte Königreich - ,emotional and misguided" sei. ${ }^{25}$

Insgesamt gehen die Ergebnisse der Analyse der Schuldvermeidungspolitik der Europäischen Kommission in der EU-Sozialleistungspolitik mit unseren theoretischen Erwartungen konform. Die hybride EU-Autorität in der EU-Sozialleistungspolitik ermöglichte es den Mitgliedstaaten, in hohem Maße Verantwortung auf die EU-Ebene zu schieben, weshalb die Notwendigkeit für die EU-Kommission groß war, aus dem Fokus der Öffentlichkeit zu gelangen. Zugleich verfügte sie jedoch auch über ausreichend Möglichkeiten, um die Schuld zurück auf die Mitgliedstaaten zu schieben und reagierte deshalb mit einer Strategie des Attackierens.

\footnotetext{
21 Viviane Reding, EU-Kommissarin, zit. Waterfield (2013).

22 Viviane Reding, EU-Kommissarin, zit. Lynch (2013); Herv. d. Autoren.

23 Vera Jourov, EU-Kommissarin, zit. Nardelli et al. (2015).

24 Margaritis Schinas, Sprecher der EU-Kommission, zit. Irish Times (2014).

25 László Andor, EU-Kommissar, zit. Vogel (2014).
} 


\section{Schluss: IOs als Schuldvermeider}

Die Analyse der drei Fälle von umstrittenen EU-Migrationspolitiken unterstützt unsere These, dass die Autorität der jeweiligen IO bedingt, mit welcher Schuldvermeidungsstrategie ihre führenden RepräsentantInnen auf Schuldzuweisungen der Mitgliedstaaten reagieren: In der Asylpolitik, die durch intergouvernementale Autorität geprägt ist, wählte die EU-Kommission die Strategie des Ignorierens; im Fall der Grenzschutzpolitik, in der die Autorität eher supranational ist, wählte die Kommission eine Strategie des Verschleierns; und hinsichtlich der umstrittenen Sozialleistungspolitik, bei der sich die nationale und europäische Ebene die Autorität teilen, wählte die Kommission eine Strategie des Attackierens. Weit davon entfernt, ihren Mitgliedstaaten nur passiv als ,,blame taker“ zu dienen, prägt die EU-Kommission in den untersuchten Fällen die ,blame games“ in der Öffentlichkeit als „,blame avoider" aktiv mit.

Aber lassen sich diese Ergebnisse von der EU auch auf andere IOs übertragen? Im Rahmen unseres Most-similar-case-Designs lässt sich dies schwer sagen. Denn das Design zielt auf interne und nicht auf externe Validität ab. Um letzteres abzuschätzen, wäre ein Most-different-case-Design oder auch ein Hard-case-Design notwendig. Trotzdem verleihen die empirischen Befunde in den untersuchten Fällen unserer Theorie zumindest vorläufig Plausibilität. Sie sind somit als Appell an die Forschung zu verstehen, sich mit ihrer bisher zumeist unhinterfragten Annahme von IOs als „,blame taker“ vermehrt kritisch auseinanderzusetzen und systematisch zu diskutieren, inwieweit IOs - wie fast alle politischen Akteure - auch als ,blame avoider" zu verstehen sind. Dabei wäre dann auch systematisch zu prüfen, ob sich unsere Theorie zum einen innerhalb der EU auch jenseits des Politikfelds der Migration bewährt und zum anderen auch auf IOs jenseits der EU anwendbar ist.

Derartige Forschung scheint umso notwendiger, als unsere Theorie erwarten würde, dass aufgrund ihres fortschreitenden Autoritätszuwachses (Zürn et al. 2012; Genschel und Zangl 2014; Hooghe und Marks 2015) IOs immer weniger mitgliedstaatliche Schuldzuweisungen einfach ignorieren werden, um stattdessen Schuld vermehrt zu verschleiern oder sogar ihre Mitgliedstaaten durch eigene Schuldzuweisungen zu attackieren. Ein aktuelles Beispiel dafür bietet die WHO, die auf Anschuldigungen der USA, in der Coronakrise versagt zu haben, ganz offen ihre Mitgliedstaaten, insbesondere die USA, für ihre mangelhafte Coronapolitik kritisierte. Ein anderes Beispiel ist die Organisation für Sicherheit und Zusammenarbeit in Europa (OSZE), die die Kritik der Ukraine, ihre Mission zur Überwachung des Minsker-Abkommens würde Russland bevorzugen, nicht nur zurückgewiesen hat, sondern ihrerseits die Ukraine der mangelnden Kooperation beschuldigte. Und ein letztes Beispiel bietet die Internationale Atomenergie-Organisation (IAEA), welche auf Vorwürfe der USA, die Einhaltung des „Atomdeals“ mit dem Iran nicht angemessen überwacht zu haben, ihrerseits die USA der Destabilisierung des Abkommens bezichtigte. Insgesamt gibt es also durchaus Indizien, dass mit ihrem Autoritätszuwachs IOs auch jenseits der EU nicht mehr nur als ,blame taker“, sondern auch als „,blame avoider“ auftreten. 
Förderung This work was supported by the Deutsche Forschungsgemeinschaft (grant number 391007015)

Funding Open Access funding provided by Projekt DEAL.

Open Access Dieser Artikel wird unter der Creative Commons Namensnennung 4.0 International Lizenz veröffentlicht, welche die Nutzung, Vervielfältigung, Bearbeitung, Verbreitung und Wiedergabe in jeglichem Medium und Format erlaubt, sofern Sie den/die ursprünglichen Autor(en) und die Quelle ordnungsgemäß nennen, einen Link zur Creative Commons Lizenz beifügen und angeben, ob Änderungen vorgenommen wurden.

Die in diesem Artikel enthaltenen Bilder und sonstiges Drittmaterial unterliegen ebenfalls der genannten Creative Commons Lizenz, sofern sich aus der Abbildungslegende nichts anderes ergibt. Sofern das betreffende Material nicht unter der genannten Creative Commons Lizenz steht und die betreffende Handlung nicht nach gesetzlichen Vorschriften erlaubt ist, ist für die oben aufgeführten Weiterverwendungen des Materials die Einwilligung des jeweiligen Rechteinhabers einzuholen.

Weitere Details zur Lizenz entnehmen Sie bitte der Lizenzinformation auf http://creativecommons.org/ licenses/by/4.0/deed.de.

\section{Literatur}

Abbott, Kenneth W., Philipp Genschel, Duncan Snidal, und Bernhard Zangl. 2020. Competence-control theory: the challenge of governing through intermediaries. In Governor's Dilemma: Indirect governance beyond principals and agents, Hrsg. Kenneth W. Abbott, Philipp Genschel, Duncan Snidal, und Bernhard Zangl, 3-38. Oxford: Oxford University Press.

Adam, Silke, und Barbara Pfetsch. 2009. Europa als Konflikt in nationalen Medien: Zur Politisierung der Positionen in der Integrationsdebatte. In Politik in der Mediendemokratie, 1. Aufl., Politische Vierteljahresschrift. Sonderheft, Bd. 42, Hrsg. Frank Marcinkowski, Barbara Pfetsch, 559-584. Wiesbaden: VS.

Alcañiz, Isabella, und Timothy T. Hellwig. 2011. Who's to blame? The distribution of responsibility in developing democracies. British Journal of Political Science 41(2):389-411. https://doi.org/10.1017/ S0007123409990317.

Baume, Maïa de la. 2015. Tusk says EU reputation under threat: The European Council president tells MEPs that a lack of solidarity on migration is giving the Union a bad name. Politico Europe. 6. Oktober 2015.

Boin, Arjen, Paul 't Hart, und Allan McConnell. 2009. Crisis exploitation: political and policy impacts of framing contests. Journal of European Public Policy 16(1):81-106. https://doi.org/10.1080/ 13501760802453221.

Brändström, Annika, und Sanneke Kuipers. 2003. From 'normal incidents' to political crises: understanding the selective politicization of policy failures. Government and Opposition 38(3):279-305. https:// doi.org/10.1111/1477-7053.t01-1-00016.

Buchanan, Allen, und Robert O. Keohane. 2006. The legitimacy of global governance institutions. Ethics \& International Affairs 20(4):405-437. https://doi.org/10.1111/j.1747-7093.2006.00043.x.

Capelos, Tereza, und Johannes Wurzer. 2009. United front: blame management and scandal response tactics of the united nations. Journal of Contingencies and Crisis Management 17(2):75-94. https://doi. org/10.1111/j.1468-5973.2009.00567.x.

Der Standard. 2015. Erste Priorität ist, das Sterben im Meer zu beenden. Der Standard. 22. April 2015.

Dingwerth, Klaus, Henning Schmidtke, und Tobias Weise. 2019a. The rise of democratic legitimation: why international organizations speak the language of democracy. European Journal of International Relations https://doi.org/10.1177/1354066119882488.

Dingwerth, Klaus, Antonia Witt, Ina Lehmann, Ellen Reichel, und Tobias Weise. 2019b. International organizations under pressure. Legitimating global governance in challenging times. Oxford: Oxford University Press.

Ecker-Ehrhardt, Matthias. 2018a. International organizations "going public"? An event history analysis of public communication reforms 1950-2015. International Studies Quarterly 62(4):723-736. https:// doi.org/10.1093/isq/sqy025. 
Ecker-Ehrhardt, Matthias. 2018b. IO public communication and discursive inclusion: how the UN reported the Arms Trade Treaty process to a global audience. Journal of International Relations and Development https://doi.org/10.1057/s41268-018-0143-3.

Ecker-Ehrhardt, Matthias. 2018c. Self-legitimation in the face of politicization: why international organizations centralized public communication. The Review of International Organizations 13(4):519-546. https://doi.org/10.1007/s11558-017-9287-y.

Europäische Kommission. 2017. White paper on the future of europe. Reflections and scenarios for the EU27 by 2025. https://ec.europa.eu/commission/sites/beta-political/files/white_paper_on_the_ future_of_europe_en.pdf. Zugegriffen: 21. Apr. 2020.

Frankfurter Allgemeine Zeitung (FAZ). 2013. Barroso: Der Notstand von Lampedusa ist ein europäischer. Frankfurter Allgemeine Zeitung. 10. Oktober 2013.

Gattermann, Katjana. 2018. Mediated personalization of executive European Union politics: examining patterns in the Broadsheet coverage of the European Commission, 1992-2016. The international journal of press/politics 23(3):345-366. https://doi.org/10.1177/1940161218779231.

Genschel, Philipp, und Bernhard Zangl. 2014. State transformations in OECD countries. Annual Review of Political Science 17(1):337-354. https://doi.org/10.1146/annurev-polisci-061312-113943.

Gerhards, Jürgen, Anke Offerhaus, und Jochen Roose. 2005. Die Europäische Union und die massenmediale Attribution von Verantwortung. Projektskizze einer länder-, zeit- und medienvergleichenden Untersuchung. Berliner Studien zur Soziologie Europas, Bd. 3. Berlin: Lehrstuhl für Makrosoziologie der Freien Universität Berlin.

Gerhards, Jürgen, Anke Offerhaus, und Jochen Roose. 2009. Wer ist verantwortlich? Die Europäische Union, ihre Nationalstaaten und die massenmediale Attribution von Verantwortung für Erfolge und Misserfolge. In Politik in der Mediendemokratie Politische Vierteljahresschrift Sonderhefte, Bd. 42, Hrsg. Frank Marcinkowski, Barbara Pfetsch, 529-558. Wiesbaden: VS.

Gramberger, Marc R. 1997. Die Öffentlichkeitsarbeit der Europäischen Kommission 1952-1996. PR zur Legitimation von Integration? Nomos Universitätsschriften: Politik, Bd. 75. Baden-Baden: Nomos.

Gramberger, Marc R., und Ingrid Lehmann. 1995. UN und EU: Machtlos im Kreuzfeuer der Kritik. Publizistik 40(2):186-204.

Greenhill, Brian. 2020. How can international organizations shape public opinion? Analysis of a pair of survey-based experiments. The Review of International Organizations 15(1):165-188. https://doi.org/ 10.1007/s11558-018-9325-4.

Greuter, Nicole. 2014. Accountability without election. The attribution of responsibility in the financial crisis 2007-2010. Schriftenreihen politischer Kommunikation und demokratischer Öffentlichkeit, Bd. 10. Baden-Baden: Nomos.

Gronau, Jennifer, und Henning Schmidtke. 2016. The quest for legitimacy in world politics-international institutions' legitimation strategies. Review of International Studies 42(3):535-557. https://doi.org/ 10.1017/S0260210515000492.

Heinkelmann-Wild, Tim, und Bernhard Zangl. 2019. Multilevel blame games: blame-shifting in the European Union. Governance https://doi.org/10.1111/gove.12459.

Heinkelmann-Wild, Tim, Lisa Kriegmair, Berthold Rittberger, und Bernhard Zangl. 2020a. Divided they fail: the politics of wedge issues and Brexit. Journal of European Public Policy 27(5):723-741. https://doi.org/10.1080/13501763.2019.1683058.

Heinkelmann-Wild, Tim, Lisa Kriegmair, und Berthold Rittberger. 2020b. The EU multi-level system and the Europeanization of domestic blame games. Politics and Governance 8(1):85-94. https://doi.org/ 10.17645/pag.v8i1.2522.

Heinkelmann-Wild, Tim, Berthold Rittberger, und Bernhard Zangl. 2018. The European blame game: explaining public responsibility attributions in the European Union. In Political mistakes and policy failures in international relations, Hrsg. Andreas Kruck, Kai Oppermann, und Alexander Spencer, 171-189. Basingtoke: Palgrave Macmillan.

Heupel, Monika, und Bernhard Zangl. 2004. Von ,,alten“ und „,neuen“ Kriegen - Zum Gestaltwandel kriegerischer Gewalt. Politische Vierteljahresschrift 45(3):346-369. https://doi.org/10.1007/s11615-0040058-z.

Hobolt, Sara B., und James Tilley. 2014. Blaming Europe? Responsibility without accountability in the European Union. Oxford: Oxford University Press.

Hoesch, Kirsten. 2003. Kontinuität und Wandel in der Kommunikationsstrategie der EU-Kommission. Osnabrïck: Der Andere Verlag.

Hood, Christopher. 2011. The blame game: spin, bureaucracy, and self-preservation in government. Princeton: Princeton University Press. 
Hood, Christopher, Will Jennings, und Paul Copeland. 2016. Blame avoidance in comparative perspective: reactivity, staged retreat and efficacy. Public Administration 94(2):542-562. https://doi.org/10.1111/ padm.12235.

Hooghe, Liesbet, und Gary Marks. 2015. Delegation and pooling in international organizations. The Review of International Organizations 10(3):305-328. https://doi.org/10.1007/s11558-014-9194-4.

Irish Times. 2014. Cameron delivers EU exit warning. Irish Examiner. 29. November 2014.

Jankauskas, Vytautas, und Steffen Eckhard. 2019. International bureaucracies as strategic actors: how the better regulation reform strengthens the European commission. Politische Vierteljahresschrift 60(4):681-699. https://doi.org/10.1007/s11615-019-00189-3.

Kirchgaessner, Stephanie, Ian Traynor und Patrick Kingsley. 2015. Two more migrant boats issue distress calls in Mediterranean. The Guardian. 20. April 2015.

Kleinen-von Königslöw, Katharina, Senja Post, und Mike S. Schäfer. 2019. How news media (de-)legitimize national and international climate politics-A content analysis of newspaper coverage in five countries. International Communication Gazette 81(7):518-540. https://doi.org/10.1177/ 1748048518825092 .

Koopmans, Ruud. 2007. Who inhabits the European public sphere? Winners and losers, supporters and opponents in Europeanised political debates. European Journal of Political Research 46(2):183-210. https://doi.org/10.1111/j.1475-6765.2006.00691.x.

Kumlin, Staffan. 2011. Claiming blame and giving credit? Unintended effects of how government and opposition frame the Europeanization of welfare. European Union Politics 12(4):575-595. https:// doi.org/10.1177/1465116511417296.

Kunda, Ziva. 1990. The case for motivated reasoning. Psychological Bulletin 108(3):480-498. https://doi. org/10.1037/0033-2909.108.3.480.

Lavenex, Sandra. 2015. Justice and home affairs: institutional change and policy continuity. In Policy-making in the European Union, 7. Aufl., Hrsg. Helen Wallace, Mark A. Pollack, und Alasdair R. Young, 367-387. Oxford: Oxford University Press.

Leibfried, Stephan. 2015. Social policy. Left to judges and the markets? In Policy-making in the European Union, 7. Aufl., Hrsg. Helen Wallace, Mark A. Pollack, und Alasdair R. Young, 263-292. Oxford: Oxford University Press.

León, Sandra, Ignacio Jurado, und Amuitz Garmendia Madariaga. 2018. Passing the buck? Responsibility attribution and cognitive bias in multilevel democracies. West European Politics 41(3):660-882. https://doi.org/10.1080/01402382.2017.1405325.

Lynch, Suzanne. 2013. UK calls for overhaul of EU rules on migrants; Home secretary proposes immigration cap and change to law on free movement. The Irish Times. 6. Dezember 2013.

Nardelli, Alberto, Ian Traynor, Leila Haddou und Rowena Mason. 2015. Fortress mentality on EU migration creates xenophobia, warns Italian PM; Matteo Renzi says defensive attitude in debate about freedom of movement opens the door to right-wing nationalism. The Guardian. 19. Januar 2015.

Palmeri, Tara. 2015. EU vows beefed-up response to migrant crisis: European Council President Donald Tusk calls for an emergency summit of EU leaders in Brussels on Thursday. Politico Europe. 20. April 2015.

Panichi, James. 2015. Low-key speech on asylum points to rift between member states. European Voice. 15. Januar 2015.

Przeworski, Adam, und Henry Teune. 1982. The logic of comparative social inquiry. Malabar: R.E. Krieger Publishing Company.

Rauh, Christian, und Michael Zürn. 2019. Authority, politicization, and alternative justifications: endogenous legitimation dynamics in global economic governance. Review of International Political Econo$m y$ https://doi.org/10.1080/09692290.2019.1650796.

Rauh, Christian, Bart Joachim Bes, und Martijn Schoonvelde. 2020. Undermining, defusing or defending European integration? Assessing public communication of European executives in times of EU politicisation. European Journal of Political Research 59(1):397-423. https://doi.org/10.1111/1475-6765. 12350.

Rittberger, Berthold, Helena Schwarzenbeck, und Bernhard Zangl. 2017. Where does the buck stop? Responsibility attributions in complex international institutions. Journal of Common Market Studies 55(4):909-924. https://doi.org/10.1111/jcms.12524.

Roose, Jochen, Franziska Scholl, Moritz Sommer, und Angelos Loukakis. 2017. Debating responsibility on the Eurozone crisis in traditional media newspaper reporting in Greece and Germany under a soial media lens. In Social media and European politics. Rethinking power and legitimacy in the digital era, Hrsg. Mauro Barisione, Asimina Michailidou, 267-290. Basingtoke: Palgrave Macmillan. 
Roose, Jochen, Franziska Scholl, und Moritz Sommer. 2018. Verantwortungszuschreibung in der Eurozonen-Krise. Das Kommunikationsverhalten von Politik und Zivilgesellschaft im deutsch-griechischen Vergleich. In Europas Zivilgesellschaft in der Wirtschafts- und Finanzkrise: Protest, Resilienz und Kämpfe um Deutungshoheit, Hrsg. Jochen Roose, Moritz Sommer, und Franziska Scholl, 43-73. Wiesbaden: VS.

Schlipphak, Bernd, und Oliver Treib. 2017. Playing the blame game on Brussels: the domestic political effects of EU interventions against democratic backsliding. Journal of European Public Policy 24(3):352-365. https://doi.org/10.1080/13501763.2016.1229359.

Schwarzenbeck, Helena. 2015. Politische Verantwortung - verdeckt, verschoben, verschleiert? Der Einfluss von Governance-Design auf öffentliche Verantwortungsattribution. Inaugural-Dissertation zur Erlangung des Doktorgrades der Philosophie. München: Ludwig-Maximilians-Universität München.

Sommer, Moritz. 2019. Blame-shifting in times of permanent austerity: evidence from Spain and Greece in the Eurozone crisis. Journal of Common Market Studies https://doi.org/10.1111/jcms.12926.

Sommer, Moritz, Jochen Roose, Franziska Scholl, und Dimitris Papanikolopoulos. 2016. The Eurozone crisis and party conflics in Greece and Germany: discursive struggles about responsibility. In Europe's crisis: the conflict theoretic perspective, Hrsg. Tim Krieger, Bernhard Neumärker, und Diana Panke, 87-110. Baden-Baden: Nomos.

Squatrito, Theresa, Magnus Lundgren, und Thomas Sommerer. 2019. Shaming by international organizations: mapping condemnatory speech acts across 27 international organizations, 1980-2015. Cooperation and Conflict 54(3):356-377. https://doi.org/10.1177/0010836719832339.

Straub, Dominik. 2015. Neuerlich hunderte Flüchtlinge ertrunken. Der Standard. 20. April 2015.

Tallberg, Jonas, und Michael Zürn. 2019. The legitimacy and legitimation of international organizations: introduction and framework. The Review of International Organizations 14(4):581-606. https://doi. org/10.1007/s11558-018-9330-7.

Tallberg, Jonas, Karin Bäckstrand, und Jan Aart Scholte. 2018. Introduction: legitimacy in global governance. In Legitimacy in global governance: sources, processes, and consequences, Hrsg. Jonas Tallberg, Karin Bäckstrand, und Jan Aart Scholte, 3-19. Oxford: Oxford University Press.

Traber, Denise, Martijn Schoonvelde, und Gijs Schumacher. 2019. Errors have been made, others will be blamed: Issue engagement and blame shifting in Prime Minister speeches during the economic crisis in Europe. European Journal of Political Research 59(1):45-67. https://doi.org/10.1111/1475-6765. 12340.

Traynor, Ian. 2015. EU to launch military operations against migrant-smugglers in Libya. The Guardian. 21. April 2015.

Vasilopoulou, Sofia, Daphne Halikiopoulou, und Theofanis Exadaktylos. 2014. Greece in crisis: austerity, populism and the politics of blame. Journal of Common Market Studies 52(2):388-402. https://doi. org/10.1111/jcms.12093.

Vogel, Toby. 2014. Fanning the flames of the migration debate. European Voice. 16. Januar 2014.

Waterfield, Bruno. 2013. Don't blame EU for your generous welfare system, Britain told. The Daily Telegraph. 6. Dezember 2013.

Weaver, R. Kent. 1986. The politics of blame avoidance. Journal of Public Policy 6(4):371-398.

Weaver, R. Kent. 2018. The nays have it: how rampant blame generating distorts American policy and politics. Political Science Quarterly 133(2):259-289. https://doi.org/10.1002/polq.12771.

Weaver, Matthew. 2015a. Refugee crisis: Juncker unveils EU quota plan. The Guardian. 9. September 2015.

Weaver, Matthew. 2015b. Refugee crisis: EU splits exposed at emergency summit. The Guardian. 24. September 2015.

Wilson, Traci L., und Sara B. Hobolt. 2015. Allocating responsibility in multilevel government systems: voter and expert attributions in the European Union. The Journal of Politics 77(1):102-113. https:// doi.org/10.1086/678309.

Zaum, Dominik (Hrsg.). 2013. Legitimating international organizations. Oxford: Oxford University Press.

Zürn, Michael, Martin Binder, und Matthias Ecker-Ehrhardt. 2012. International authority and its politicization. International Theory 4(1):69-106. https://doi.org/10.1017/S1752971912000012. 